\title{
Tiền tố và hậu tố của nhận diện thương hiệu: Nghiên cứu thực nghiệm thương hiệu điện thoại di động tại Việt Nam
}

\section{Antecedents and outcome of consumer-brand identification: An empirical study of smartphones' brands in Vietnam}

\author{
Lý Mỹ Hằng ${ }^{1}$, Nguyễn Thị Mai Trang ${ }^{2 *}$ \\ ${ }^{1}$ Công ty Cổ phần We Food, Việt Nam \\ ${ }^{2}$ Trường Đại học Kinh tế Thành phố Hồ Chí Minh, Việt Nam \\ *Tác giả liên hệ, Email: trangntm@ueh.edu.vn
}

THÔNG TIN

DOI: $10.46223 / \mathrm{HCMCOUJS}$. econ.vi.16.3.577.2021

Ngày nhận: 09/07/2020

Ngày nhận lại: 26/12/2020

Duyệt đăng: 31/12/2020

\section{Tù khóa:}

lý thuyết nhận dạng xã hội; nhận diện thương hiệu từ người tiêu dùng; sự khác biệt thương hiệu; uy tín thương hiệu

\section{Keywords:}

social identity theory; consumer-brand identification; brand distinctiveness; brand prestige

\section{TÓM TÁT}

Mục đích của nghiên cứu này nhằm xem xét mức độ ảnh hưởng của một số nhân tố: sự khác biệt thương hiệu, uy tín thương hiệu, lợi ích xã hội thương hiệu và sự thân thiện thương hiệu đến nhận diện thương hiệu, và sau đó đến hành vi truyền miệng. Mô hình cấu trúc tuyến tính (SEM) được sử dụng để kiểm định mối quan hệ trên thông qua 269 người tiêu dùng đã mua và sử dụng điện thoại di động tại Thành phố Hồ Chí Minh, Việt Nam. Kết quả nghiên cứu chỉ ra rằng bốn tiền tố sự khác biệt thương hiệu, uy tín thương hiệu, lợi ích xã hội thương hiệu và sự thân thiện thương hiệu có mối quan hệ cùng chiều với nhận diện thương hiệu. Ngoài ra, nhận diện thương hiệu có tương quan cùng chiều với sự truyền miệng của người tiêu dùng.

ABSTRACT
This study examines the impacts of brand distinctiveness,
brand prestige, brand social benefits, and brand warmth on
consumer-brand identification, which in turn influences word of
mouth. Structural Equation Modeling (SEM) was used to test
these relationships, using a sample of 269 mobile phone users in
Ho Chi Minh City. The results show that the four antecedents,
such as brand distinctiveness, brand prestige, brand social
benefits, and brand warmth, positively affect consumer brand
identification. In addition, consumer brand identification has a
positive influence on word of mouth.

\section{Giới thiệu}

Thị trường smartphone Việt là cuộc chiến cạnh tranh khốc liệt giữa các thương hiệu nội địa và nước ngoài, đòi hỏi các hãng điện thoại phải xây dựng được sự nhận diện thương hiệu của người tiêu dùng. Tính riêng smartphone, các thương hiệu lớn tại Việt Nam hiện là Samsung, Apple, Oppo đang chiếm tới trên dưới $80 \%$ thị phần về sản lượng và xấp xỉ $70 \%$ thị phần về giá trị, $20 \%$ thị phần doanh số còn lại với khoảng $30 \%$ giá trị thị trường dành cho hàng chục thương hiệu cũ mới, cả nội địa và nước ngoài (Huu Tuan, 2020). Thị trường điện thoại di động tràn ngập các thương hiệu Việt như: FPT, Mobiistar, Masstel cùng với những thương 
hiệu ngoại như Samsung, Apple, Oppo, Sony, Asus, Huawei. Cụ thể, theo số liệu từ hãng GfK, người Việt chi 19.8 nghìn tỉ đồng mua 3.64 triệu smartphone trong ba tháng đầu năm 2017. Con số này tăng so với 17.8 nghìn tỷ đồng cho 3.47 triệu smartphone cùng kỳ năm 2017 (Thanh Truc, 2018). Sức mạnh thương hiệu đã được các nhà nghiên cứu hàn lâm và thực tiễn công nhận rộng rãi (Kumar \& Kaushilk, 2018; Nyadzayo, Leckie, \& Johnson, 2020) đặc biệt là trong thị trường điện thoại di động. Thương hiệu giúp người sở hữu nó thể hiện và đóng vai trò quan trọng trong hành vi tiêu dùng của họ (Kumar, 2020; Kumar \& Kaushilk, 2018).

Trong bối cảnh môi trường cạnh tranh khốc liệt, việc xây dựng mối quan hệ với khách hàng là điều kiện đảm bảo sự thành công của công ty (Bhattacharya \& Sen, 2003). Cơ sở để khách hàng xây dựng mối quan hệ với doanh nghiệp là thông qua sự hiểu biết về thương hiệu của doanh nghiệp, nói cách khác, là doanh nghiệp làm thế nào để khách hàng gắn bó và muốn xây dựng mối quan hệ lâu dài với doanh nghiệp là thông qua thương hiệu (Coelho, Rita, \& Santos, 2018). Do đó, nhận diện thương hiệu từ khách hàng là chủ đề quan trọng trong quản trị thương hiệu trong công ty và được các nhà hàn lâm và doanh nghiệp quan tâm nhiều (e.g., Bhattacharya \& Sen, 2003; Coelho et al., 2018; Han, Ekinci, Chen, \& Park, 2020; Lam, Ahearne, Mullins, Hayati, \& Schillewaert, 2013; Sauer, Ratneshwar, \& Sen, 2012; Yeh, Wang, \& Yieh, 2016).

Sức mạnh thương hiệu đã được các nhà nghiên cứu hàn lâm và thực tiễn công nhận rộng rãi (Ghorbanzadeh, Saeednia, \& Rahehagh, 2020; Kumar, 2020; Nyadzayo et al., 2020) đặc biệt là trong thị trường điện thoại di động. Thương hiệu giúp người sử dụng thể hiện địa vị của họ trong xã hội (Ghorbanzadeh et al., 2020). Ví dụ: những thương hiệu nổi tiếng như Apple, Samsung, Oppo, etc., đã đi vào cuộc sống của con người rất gần gũi thông qua việc gắn bó tình cảm với thương hiệu này (Huu Tuan, 2020). Sở dĩ, những thương hiệu này đã chiếm được vị trí trong lòng người tiêu dùng là do người tiêu dùng đánh giá cao những gì mà thương hiệu trên cung cấp như sự khác biệt thương hiệu, uy tín thương hiệu, lợi ích xã hội mà thương hiệu đem lại, cũng như tính thân thiện của thương hiệu mang lại cho khách hàng. Vì lý do này, mối quan hệ giữa người tiêu dùng và thương hiệu trong thập kỷ qua đã thu hút được sự quan tâm của nhà nghiên cứu hàn lâm và thực tiễn (Coelho et al., 2018). Việc hiểu rõ mối quan hệ giữa thương hiệu và người tiêu dùng rất quan trọng đối với nhà tiếp thị vì ảnh hưởng của mối quan hệ này đến lợi nhuận của công ty đã được chứng minh thông qua cam kết với thương hiệu (trung thành với thương hiệu, truyền miệng tích cực (Acharya, 2020). Đó là lý do tại sao mối quan hệ giữa con người và thương hiệu cần nghiên cứu trong lĩnh vực tiếp thị (Rather, Tehseen, Itoo, \& Parrey, 2019). Về một mặt nào đó, đây vẫn còn là một lĩnh vực thách thức cho các nhà tiếp thị nói chung và nhà tiếp thị điện thoại thông minh nói riêng. Do đó, nhà nghiên cứu cần xem xét những yếu tố mang tính cảm xúc như sự khác biệt thương hiệu, uy tín của thương hiệu, lợi ích xã hội mà thương hiệu mang đến cho người sử dụng, và tính thân thiện của thương hiệu để xây dựng mối quan hệ gắn bó giữa thương hiệu và người tiêu dùng. Vì vậy, việc nghiên cứu các tiền tố đối với việc nhận dạng thương hiệu rất cần thiết để thực hiện nghiên cứu. Vì nhận dạng thương hiệu có ảnh hưởng tích cực đến lòng trung thành thương hiệu, ý định mua lại, đánh giá tích cực từ khách hàng (Kuenzel \& Halliday, 2008; Sauer et al., 2012). Nghiên cứu cũng xem xét mối quan hệ giữa tính nhận diện thương hiệu từ người tiêu dùng và tính truyền miệng.

\section{Cơ sở lý thuyết}

\subsection{Nhận diện thưong hiệu từ người tiêu dùng}

Lý thuyết nhận dạng xã hội (Social Identity Theory - SIT) mô tả khái niệm nhận diện xã hội và khởi điểm đây là một khái niệm quan trọng trong hành vi tổ chức (Lam et al., 2013; Tajfel 
\& Turner, 1986). Lý thuyết này cho rằng con người thường vượt lên trên bản sắc cá nhân của mình để phát triển bản sắc xã hội (Bhattacharya \& Sen, 2003). Dựa trên SIT, nhận dạng thương hiệu từ người tiêu dùng giải thích tại sao người tiêu dùng có mối liên hệ với công ty (Tajfel \& Turner, 1986). Bhattacharya và Sen (2003) cho rằng lý thuyết nhận diện xã hội có đóng góp hữu ích đối với tiếp thị, khách hàng thể hiện cá nhân bằng cách xác định và liên kết với các thương hiệu phản ánh và củng cố bản sắc cá nhân của họ. Điều này có thể giải thích qua mục đích khi khách hàng mua một thương hiệu nào đó, rồi sở hữu và tiêu dùng thương hiệu đó nhằm giúp khách hàng khác với người khác trong xã hội. Do vậy, thương hiệu có khả năng thể hiện, thông tin và truyền đạt bản sắc người tiêu dùng mong muốn (Bhattacharya $\&$ Sen, 2003). Trong nghiên cứu này, chúng tôi sử dụng định nghĩa của Elbedweihy và Jayawardhena (2014), nhận diện thương hiệu của khách hàng là một trạng thái nhận thức của người tiêu dùng về sự thống nhất, gắn bó và hòa hợp của cá nhân với một thương hiệu cụ thể nào đó.

Sự nhận dạng của khách hàng được xem là quá trình tâm lý mà quá trình này có ảnh hưởng sự sẵn lòng khách hàng trong việc hình thành mối quan hệ dài hạn và gắn bó của họ với công ty và thương hiệu (Lam, Ahearne, $\mathrm{Hu}, \&$ Schillewaert, 2010). Nhiều nghiên cứu đã tập trung nghiên cứu các tiền tố ảnh hưởng đến sự nhận dạng khách hàng - thương hiệu. Ví dụ: phù hợp giá trị (value congruity) (Tuškej, Golob, \& Podnar, 2013); chất lượng cảm nhận (perceived quality) và sự phù hợp của thương hiệu (self-brand congruity) (Lam et al., 2010). Nghiên cứu này sẽ tập trung nghiên cứu những tiền tố (như sự khác biệt thương hiệu, uy tín thương hiệu, lợi ích xã hội của thương hiệu, và sự thân thiện của thương hiệu) ảnh hưởng đến sự nhận dạng khách hàng - thương hiệu. Nghiên cứu trước đây đã xem xét về các hậu tố của sự nhận dạng khách hàng - thương hiệu, đó là hành vi của khách hàng như dự định mua lại, trung thành thương hiệu, và truyền miệng (Kuenzel \& Halliday, 2008; Sauer et al., 2012). Nghiên cứu này xem xét yếu tố truyền miệng (WOM) là kết quả (hậu tố) của sự nhận dạng khách hàng - thương hiệu, WOM là việc thông tin sản phẩm từ một khách hàng đến khách hàng khác. Điều này cho thấy, thông tin truyền miệng là một công cụ quan trọng của tiếp thị truyền thông trong công ty vì WOM đóng vai trò quan trọng trong việc hình thành thái độ và hành vi của khách hàng.

\subsection{Sụ khác biệt thưong hiệu}

Theo lý thuyết nhận dạng xã hội, con người luôn có nhu cầu chứng minh chúng ta thuộc vào nhóm nào đó và cố gắng phân biệt mình với người khác (Tajfel \& Turner, 1986) với mục đích giúp chúng ta cảm thấy bản thân mình tốt hơn người khác. Nói cách khác, để thỏa mãn nhu cầu giống với người khác nhưng có điểm độc đáo cá nhân riêng, người tiêu dùng giải quyết nhu cầu trên bằng cách tham gia vào các nhóm đáp ứng cả hai nhu cầu trên (Brewer, 1991).

Do đó, sự khác biệt thương hiệu là tiền đề then chốt dẫn đến mong muốn có cùng đặc điểm với thương hiệu đó của người tiêu dùng (Berger \& Heath, 2007). Người tiêu dùng thường khẳng định bản thân cá nhân của mình thông qua việc tiêu dùng các sản phẩm có thương hiệu hơn là các sản phẩm được sản xuất và tiêu dùng hàng loạt (Thompson, Rindfleisch, \& Arsel, 2006). Các doanh nghiệp trên thị trường luôn cố gắng tìm mọi cách để định vị sản phẩm hoặc dịch vụ của mình làm sao có một kiểu dáng hoặc phong cách khác biệt với đối thủ cạnh tranh (Keller, 2003). Sự khác biệt này sẽ tạo cho các doanh nghiệp lợi thế tiếp thị vượt trội hơn đối thủ cạnh tranh. Giữa tất cả các thương hiệu như nhau, thương hiệu nào có hình ảnh hoặc đặc tính khác biệt thì sẽ có nhiều khả năng được người tiêu dùng nhận diện hơn, với điều kiện cơ sở của sự khác biệt này được khách hàng chấp nhận trên thị trường. Các nghiên cứu tiếp thị khác cũng nhấn mạnh tầm quan trọng của sự khác biệt trong định vị thương hiệu, góp phần tạo nên thành công của một thương hiệu (Sihvonen, 2019). Sự khác biệt được định nghĩa là mức độ người tiêu dùng nhận thức được một thương hiệu khác biệt với đối thủ cạnh tranh (Zhang, Li, Ye, Qin, \& 
Zhong, 2020). Thương hiệu có thể có giá cao hơn nếu được xem là khác biệt so với đối thủ cạnh tranh. Tóm lại, sự khác biệt thương hiệu được định nghĩa là tính độc đáo của thương hiệu so với đối thủ cạnh tranh, và là một yếu tố ảnh hưởng đến việc nhận diện thương hiệu CBI. Do đó:

H1: Sư khác biệt thuoong hiệu có tác động tích cực đến sụ nhận diện thuơng hiệu của người tiêu dùng

\subsection{Uy tín thuong hiệu}

Uy tín thương hiệu có thể đại diện cho vị thế tương đối cao của sản phẩm định vị gắn liền với thương hiệu (Truong, McColl, \& Kitchen, 2009). Alden, Steenkamp, và Batra (1999) lập luận rằng người tiêu dùng có xu hướng nhận thức việc tiêu dùng các thương hiệu uy tín được xem như một tín hiệu về địa vị xã hội, sự giàu có, hoặc quyền lực vì thương hiệu uy tín không được mua thường xuyên và liên quan chặt chẽ đến hình ảnh xã hội của cá nhân. Theo Han và cộng sự (2020) thương hiệu uy tín khác với thương hiệu không uy tín theo một số cách có thể ảnh hưởng đến động cơ mua hàng của người tiêu dùng, ví dụ để nâng cao vị thế xã hội và sự thể hiện bản thân của họ. So với thương hiệu không uy tín, thương hiệu uy tín không chỉ cung cấp những lợi ích vô hình cho người tiêu dùng, mà còn tạo ra giá trị cho người tiêu dùng thông qua việc khẳng định vị thế và gây chú ý. Bên cạnh đó, nhiều nghiên cứu tiêu dùng chứng minh vai trò quyết định của việc đề cao bản thân đối với sự yêu thích của người tiêu dùng dành cho thương hiệu (Fournier, 1998; Han et al., 2020; Thomson, MacInnis, \& Park, 2005). Do đó: tiêu dùng

H2: Uy tín thuơng hiệu có tác động cùng chiều đến sụ nhận diện thuoong hiệu của nguời

\subsection{Lọi ích xã hội thương hiệu}

Một số nghiên cứu cho thấy thương hiệu là một trong những công cụ truyền tải ý nghĩa xã hội và văn hoá (Diamond et al., 2009; Thompson et al., 2006). Lợi ích xã hội được cung cấp bởi các thương hiệu gồm ba lợi ích chính. Thứ nhất, việc tiêu dùng thương hiệu được sử dụng bởi các nhóm tham khảo (các nhóm xã hội quan trọng đối với người tiêu dùng) giúp mọi người đạt được hoặc tăng cường tư cách thành viên của họ trong các nhóm này (Han et al., 2020). Thứ hai, thương hiệu là một phương tiện thiết yếu kết nối mọi người với nhau (Muniz \& O'Guinn, 2001). Một cộng đồng thương hiệu có thể được định nghĩa như một cộng đồng chuyên biệt, không giới hạn địa lý dựa trên một bộ cấu trúc quan hệ xã hội giữa những người hâm mộ một thương hiệu (Muniz \& O'Guinn, 2001). Thành viên trong các cộng đồng như vậy không chỉ gắn bó với cộng đồng, mà còn gắn bó với thương hiệu vốn là lý do tồn tại của cộng đồng đó (Cheung, Pires, \& Rosenberger, 2020). Cuối cùng, sự chia sẻ cam kết với một thương hiệu giúp người tiêu dùng kết hợp thành những phân nhóm khác biệt của xã hội (Thompson et al., 2006). Các nhóm cộng đồng thương hiệu có một cấu trúc xã hội có thứ bậc, tập hợp các niềm tin và giá trị được chia sẻ, tập hợp các trình tự và biệt ngữ độc đáo. Những lợi ích trên tạo cơ sở cho lập luận: người tiêu dùng có nhiều khả năng ủng hộ các nhãn hiệu giúp họ kết nối với các cá nhân, các nhóm, cộng đồng quan trọng.

Các nhà tiếp thị đã trở nên quan tâm hơn đến việc nghiên cứu, tổ chức và tạo điều kiện cho cộng đồng thương hiệu (McAlexander, Schouten, \& Koenig, 2002), cộng đồng được "dựa trên một tập các mối quan hệ có cấu trúc trong số những người ngưỡng mộ một thương hiệu" (Muniz \& O'Guinn, 2001, p. 412). Có nhiều lý do làm cơ sở cho sự quan tâm này, bao gồm khả năng các cộng đồng thương hiệu ảnh hưởng đến nhận thức và hành động của các thành viên (Cheung et al., 2020); phổ biến thông tin nhanh chóng (Brown, Kozinets, \& Sherry, 2003); để học hỏi đánh giá của khách hàng về các dịch vụ mới, hành động cạnh tranh, etc.; và tối đa hóa cơ hội để tương tác, cộng tác với khách hàng trung thành. Tóm lại, lợi ích xã hội của thương hiệu 
được định nghĩa là những cơ hội tương tác xã hội và lợi ích cung cấp bởi thương hiệu, lợi ích xã hội của thương hiệu được kỳ vọng là một trong những yếu tố quyết định đến CBI. Do đó:

H3: Lọi ích xã hội thwơng hiệu có tác động tích cực đến Sự nhận diện thuơng hiệu của người tiêu dùng

\subsection{Sự thân thiện thuong hiệu}

Sự thân thiện thương hiệu là mức độ mà một người tiêu dùng cảm thấy yên bình, nhiệt tâm, ấm áp hay yêu mến liên quan đến thương hiệu (Truch, 2006), cuối cùng dẫn đến cộng hưởng thương hiệu, đó là một thước đo của mối quan hệ tâm lý mà khách hàng có với thương hiệu cũng như mức độ phát sinh các hoạt động trung thành với thương hiệu. Cảm nhận của con người được chia ra hai khía cạnh chính, "sự thân thiện" và "năng lực" (Fiske, Cuddy, Glick, \& Xu, 2002). Aaker, Vohs, và Mogilner (2010) cho rằng ý định (warmth - sự thân thiện) và khả năng (competence - năng lực) là các yếu tố quan trọng cơ bản của nhận thức về thương hiệu có thể áp dụng cho mọi người và các nhóm xã hội. Ngoài ra, Fiske và cộng sự (2002) cho rằng trong nhận thức của con người, sự phân biệt lạnh - ấm là tương đối độc lập với nhận thức về chất lượng, độ tin cậy và tính năng của thương hiệu.

Mức độ một thương hiệu được cảm nhận thân thiện (cảm xúc) hơn là lạnh lùng (lý trí) là một trong những yếu tố quyết định then chốt của CBI. Thương hiệu thân thiện có khả năng thể hiện ý nghĩa trừu tượng ở mức độ cao hơn ví dụ giá trị và đạo đức của thương hiệu, chứ không phải là ý nghĩa cụ thể, thấp hơn liên quan đến các tính năng của thương hiệu. Vì vậy, Fournier (1998) cũng như Park, MacInnis, Priester, Eisingerich, và Iacobucci (2010) nhận định những thương hiệu thân thiện có xu hướng là những ứng viên mạnh mẽ và có ý nghĩa cho việc nhận diện hơn so với thương hiệu lạnh và người tiêu dùng sẽ có cảm xúc mãnh liệt về thương hiệu ấm áp cũng như vai trò của chúng trong cuộc sống của họ nhiều hơn so với thương hiệu lạnh. Zhang và cộng sự (2020) cho rằng sự thân thiện đóng vai trò nền tảng hình thành sự nhận diện thương hiệu của người tiêu dùng. Tóm lại, sự thân thiện thương hiệu là mức độ mà một người tiêu dùng cảm thấy yên bình, nhiệt tâm, ấm áp hay yêu mến liên quan đến một thương hiệu nào đó. Vì vậy:

H4: Sự thân thiện thwơng hiệu có tác động tích cực đến sụ nhận diện thuơng hiệu của người tiêu dùng

\subsection{Truyền miệng}

Truyền miệng ảnh hưởng không chỉ đến phần lớn quyết định mua hàng mà còn góp phần hình thành thái độ, hành vi người tiêu dùng. Việc nhận diện một nhóm/tổ chức có tác động tích cực đến những cống hiến của thành viên, sự hợp tác nhóm và tinh thần đóng góp cho công việc tập thể (Dutton, Dukerich, \& Harquail, 1994). Vì vậy, sự nhận diện của người tiêu dùng với một nhóm, một tổ chức hay một thương hiệu sẽ hướng họ đến những hành xử tích cực. Một số nghiên cứu cho thấy việc nhận diện của người tiêu dùng có tác động đến truyền miệng tích cực (Ahearne, Bhattacharya, \& Gruen, 2005; Bhattacharya \& Sen, 2003; Kuenzel \& Halliday, 2008). Nghiên cứu nhận diện tổ chức cho thấy rằng khi khách hàng cảm nhận mình có đặc điểm giống như đặc điểm sản phẩm của công ty nào đó, họ có xu hướng mua nhiều hơn và giới thiệu các sản phẩm của công ty này thường xuyên hơn (Ahearne et al., 2005). Hơn thế nữa, Acharya (2020) nhận thấy khi khách hàng có cùng đặc điểm với một thương hiệu và cộng đồng thương hiệu, họ có xu hướng ủng hộ và nhận xét tích cực về thương hiệu. Cheung và cộng sự (2020) cho rằng khi người tiêu dùng nhận thức một thương hiệu là sự lựa chọn duy nhất chấp nhận được vì họ gắn bó tình cảm với thương hiệu đó, họ có thể tham gia hành vi truyền miệng vì lý do thoải mái và yên tâm hoặc đơn giản bởi vì họ tin vào thương hiệu. Theo Bhattacharya và Sen (2003), người tiêu dùng có thể trở thành người bảo vệ, ủng hộ cho các công ty mà họ có cùng đặc điểm. Các hành 
vi bảo vệ có thể thay đổi tùy thuộc vào ngữ cảnh. Trong các lĩnh vực có lợi nhuận, kết quả có thể là hành vi mua sắm, truyền miệng và giới thiệu tích cực, hoặc trung thành với công ty, giới thiệu thêm khách hàng và quảng bá công ty (Bhattacharya \& Sen, 2003). Do vậy:

H5: Sự nhận diện thưong hiệu của nguời tiêu dùng có tác động tích cực đến hành vi Truyền miệng

\section{Phương pháp nghiên cứu}

Nghiên cứu này được tiến hành theo hai bước: (1) nghiên cứu định tính và (2) nghiên cứu định lượng. Nghiên cứu định tính được thực hiện thông qua kỹ thuật thảo luận tay đôi với 05 người tiêu dùng tại Thành phố Hồ Chí Minh đang sử dụng điện thoại di động. Mục đích của nghiên cứu sơ bộ nhằm điều chỉnh thang đo. Qua kết quả nghiên cứu định tính, đa số người tiêu dùng đồng ý là các câu phát biểu dễ hiểu. Nên chúng tôi tiến hành nghiên cứu định lượng. Nghiên cứu định lượng thực hiện khảo sát trực tuyến (tạo Bảng câu hỏi trên Google Docs và gửi đến đối tượng khảo sát thông qua Facebook, Zalo, Email) và gửi bảng câu hỏi (in ra giấy) và gửi cho bạn bè và người quen.

Mẫu được chọn bằng phương pháp thuận tiện (phi xác suất) với kích thước mẫu là 318 (214 bảng câu hỏi giấy và 94 qua khảo sát trực tuyến). 49 bảng trả lời bị loại do có quá nhiều ô trống hoặc chỉ chọn một câu trả lời hoặc đáp viên chưa từng mua điện thoại di động hoặc không sinh sống tại Thành phố Hồ Chí Minh. Vì vậy, kích thước mẫu cuối cùng là 269. Trong mẫu, Apple (Iphone) và Samsung là hai thương hiệu điện thoại di động được sử dụng thường xuyên nhất với tỷ lệ lần lượt là $45.7 \%$ và $33.1 \%$, kế đến là các thương hiệu Oppo $(8.2 \%)$, Nokia $(4.8 \%)$, Asus (Zenfone) (3.0\%), Sony (2.6\%), các thương hiệu khác chỉ chiếm $2.6 \%$; tỷ lệ nam là $46.1 \%$ và nữ là $53.9 \%$. Về độ tuổi, nhóm tuổi $18-22$ chiếm $18.2 \%$, nhóm tuối $23-35$ chiếm $71.4 \%$, nhóm tuổi $36-60$ và trên 60 tuổi chiếm $14 \%$. Về trình độ học vấn, nhóm có trình độ THPT chiếm 5.9\%, nhóm Trung cấp/Cao đẳng chiếm $24.9 \%$, nhóm Đại học chiếm $52 \%$, nhóm Sau đại học chiếm $17.1 \%$. Về nghề nghiệp, nhóm Nhân viên công sở chiếm $54.3 \%$; còn lại $45.7 \%$ là nghề tự kinh doanh, nội trợ và sinh viên (trong đó sinh viên chiếm 13.1\%). Về thu nhập hàng tháng, nhóm có thu nhập dưới 7 triệu đồng chiếm tỷ lệ 30.9\%, nhóm có thu nhập 7 triệu -9.9 triệu đồng chiếm $31.2 \%$, nhóm có thu nhập 10 triệu - dưới 15 triệu đồng/tháng chiếm $23.4 \%$, nhóm có thu nhập từ 15 triệu - dưới 25 triệu đồng/tháng chiếm 10.4\%, nhóm có thu nhập trên 25 triệu đồng chiếm $4.1 \%$.

Các thang đo của nghiên cứu này được kế thừa và có điều chỉnh từ những nghiên cứu trước đây. Thang đo Sự khác biệt thương hiệu (DIS) được đo bằng ba biến dựa trên nghiên cứu của Sauer và cộng sự (2012). Thang đo Uy tín thương hiệu (PRE) được đo bằng bốn biến dựa trên nghiên cứu của Sauer và cộng sự (2012). Thang đo Lợi ích xã hội của thương hiệu (SOC) cũng gồm bốn biến và dựa trên nghiên cứu của Sauer và cộng sự (2012). Thang đo Sự thân thiện thương hiệu (WAR) được đo bằng ba biến quan sát và được xây dựng dựa trên nghiên cứu của Sauer và cộng sự (2012). Thang đo Nhận diện thương hiệu của người tiêu dùng (IDE) được đo bằng 05 biến quan sát kế thừa nghiên cứu của Sauer và cộng sự (2012). Cuối cùng, thang đo Truyền miệng (WOM) được đo bằng ba biến quan sát dựa trên nghiên cứu của Kuenzel và Halliday (2008).

\section{Phân tích dữ liệu và hàm ý cho nhà quản trị}

\subsection{Kiểm định thang đo}

Hai mươi hai biến quan sát được đưa vào phân tích Cronbach's Alpha và phân tích nhân tố khám phá EFA. Sử dụng phép trích nhân tố là PAF (Principal Axis Factoring) với phép quay không vuông góc Promax. Kết quả cho thấy các nhân tố đều đạt yêu cầu giá trị hội tụ và phân 
biệt (Bảng 2). Các nhân tố sẽ được đem vào phân tích nhân tố khẳng định $\mathrm{CFA}$. Để khẳng định độ tin cậy và độ giá trị của thang đo, phương pháp CFA được áp dụng kết hợp với phương pháp ước lượng ML (Maximum Likelihood). Kết quả CFA của mô hình tới hạn cho thấy mô hình này có chi bình phương là 256.897 ( $\mathrm{p}$-value $<0.05$ ) với 194 bậc tự do, do đó chi bình phương/df = $1.324(<3)$ đạt yêu cầu. Các chỉ tiêu khác như CFI =0.979 $(\geq 0.90)$, GFI $=0.921(\geq 0.90)$, TLI = $0.975(\geq 0.90)$, RMSEA $=0.035(\leq 0.08)$ đều đạt yêu cầu. Kết luận mô hình phù hợp với dữ liệu và có tính đơn hướng. Kết quả $C F A$ của mô hình tới hạn khẳng định tính đơn hướng và giá trị hội tụ của các thang đo vì các trọng số $\left(\lambda \_i\right)$ đều cao (thấp nhất là IDE4 $=0.691$ ) và có ý nghĩa thống kê (các giá trị $\mathrm{p}<0.05$ ). Như vậy các biến quan sát dùng để đo lường cho các khái niệm đều đạt được giá trị hội tụ. Ngoài ra, độ tin cậy tổng hợp các thang đo của các khái niệm đều đạt giá trị cao (thấp nhất là DIS $=0.797$ ) và tổng phương sai trích đều lớn hơn $50 \%,($ Bảng 1$)$. Các giá trị AVE đều lớn hơn 0.5 cho tất cả các khái niệm nghiên cứu (Fornell \& Larcker, 1981). Điều này, cho thấy các khái niệm đạt giá trị phân biệt. Hay nói cách khác, các khái niệm nghiên cứu đạt giá trị phân biệt (Bảng 2).

\subsection{Kiểm định mô hình nghiên cứu và các giả thuyết}

Kết quả phân tích SEM cho thấy mô hình có 198 bậc tự do với giá trị thống kê Chi-bình phương là 259.118 (với mức ý nghĩa p-value $<0.05$ ), do đó Chi-bình phương/df $=1.309(<3)$ đạt yêu cầu. Các chỉ số CFI $=0.980(\geq 0.90)$, GFI $=0.920(\geq 0.90)$, TLI $=0.977(\geq 0.90)$, RMSEA $=0.034(\leq 0.08)$ đều đạt yêu cầu nên mô hình phù hợp với dữ liệu thị trường. Từ kết quả SEM ước lượng mô hình chuẩn hóa cho thấy các giả thuyết $\mathrm{H} 1, \mathrm{H} 2, \mathrm{H} 3, \mathrm{H} 4$ và $\mathrm{H} 5$ đều có giá trị $p$-value $<0.05$ và đều được chấp nhận (Bảng 3 ). Kết quả kiểm định các giả thuyết một lần nữa khẳng định các tiền tố Sự khác biệt thương hiệu, Uy tín thương hiệu, Lợi ích xã hội thương hiệu và Sự thân thiện thương hiệu đều có tác động tích cực đến sự Nhận diện thương hiệu của người tiêu dùng. Nhận diện thương hiệu lại có tác động tích cực đến hành vi Truyền miệng. Nghiên cứu này còn tiến hành kiểm định sự khác biệt theo giới tính. Kết quả cho thấy không có sự khác biệt trong mối quan hệ giữa tác động của bốn tiền tố đến sự nhận diện thương hiệu và hành vi truyền miệng của người tiêu dùng với cả nhóm nam và nữ (Bảng 4).

\section{Bảng 1}

Kết quả kiểm định thang đo

\begin{tabular}{lccccc}
\hline \multicolumn{1}{c}{ Khái niệm } & $\begin{array}{c}\text { Số biến } \\
\text { quan sát }\end{array}$ & $\begin{array}{c}\text { Độ tin cậy } \\
\text { tống họ̣p }\end{array}$ & $\begin{array}{c}\text { Tổng } \\
\text { phương sai } \\
\text { trích }\end{array}$ & $\begin{array}{c}\text { Trung bình } \\
\text { hệ số tải }\end{array}$ & $\begin{array}{c}\text { Giá trị } \\
\text { (hội tụ và } \\
\text { phân biệt) }\end{array}$ \\
\hline $\begin{array}{l}\text { Sự khác biệt thương hiệu } \\
\text { (DIS) }\end{array}$ & 3 & 0.797 & 0.567 & 0.753 & \\
$\begin{array}{l}\text { Uy tín thương hiệu (PRE) } \\
\begin{array}{l}\text { Lợi ích xã hội thương hiệu } \\
\text { (SOC) }\end{array}\end{array}$ & 4 & 0.867 & 0.621 & 0.787 & \\
$\begin{array}{l}\text { Sự thân thiện thương hiệu } \\
\text { (WAR) }\end{array}$ & 3 & 0.846 & 0.580 & 0.760 & Thỏa mãn \\
$\begin{array}{l}\text { Nhận diện thương hiệu } \\
\text { (IDE) }\end{array}$ & 5 & 0.851 & 0.658 & 0.809 & \\
\begin{tabular}{l} 
Truyền miệng (WOM) \\
\hline
\end{tabular} & 3 & 0.900 & 0.750 & 0.866 & \\
\hline
\end{tabular}

Nguồn: Kết quả xử lý dữ liệu từ điều tra của nhóm tác giả 


\section{Bảng 2}

Kết quả kiểm định giá trị phân biệt giữa các khái niệm nghiên cứu

\begin{tabular}{|l|c|c|c|c|c|c|c|c|}
\hline & $\mathbf{C R}$ & $\mathbf{A V E}$ & $\mathbf{1}$ & $\mathbf{2}$ & $\mathbf{3}$ & $\mathbf{4}$ & $\mathbf{5}$ & $\mathbf{6}$ \\
\hline $\begin{array}{l}\text { [1]Nhận diện TH } \\
\text { và khách hàng }\end{array}$ & 0.836 & 0.506 & $\mathbf{0 . 7 1 1 *}$ & & & & & \\
\hline [2]Tính uy tín & 0.867 & 0.621 & 0.686 & $\mathbf{0 . 7 8 8 *}$ & & & & \\
\hline [3]Lợi ích XH & 0.846 & 0.580 & 0.659 & 0.596 & $\mathbf{0 . 7 6 1 *}$ & & & \\
\hline [4]Tính thân thiện & 0.852 & 0.658 & 0.651 & 0.579 & 0.562 & $\mathbf{0 . 8 1 1 *}$ & & \\
\hline [5]Tính khác biệt & 0.797 & 0.567 & 0.611 & 0.592 & 0.431 & 0.520 & $\mathbf{0 . 7 5 3 *}$ & \\
\hline [6]Truyền miệng & 0.900 & 0.750 & 0.496 & 0.389 & 0.374 & 0.369 & 0.369 & $\mathbf{0 . 8 6 6 *}$ \\
\hline
\end{tabular}

*là căn bậc hai của AVE

Nguồn: Kết quả xử lý dữ liệu từ điều tra của nhóm tác giả

\section{Bảng 3}

Tóm tắt kết quả SEM ước lượng mô hình chuẩn hóa

\begin{tabular}{|c|c|c|c|c|c|c|}
\hline Giả thuyết & Mối quan hệ & $\mathbf{r}$ & S.E. & C.R. & P-value & Kiểm định \\
\hline H1 & $\begin{array}{l}\text { Sự khác biệt thương hiệu } \\
\rightarrow \text { Nhận diện thương hiệu }\end{array}$ & 0.224 & 0.087 & 2.976 & 0.003 & Chấp nhận \\
\hline $\mathrm{H} 2$ & $\begin{array}{l}\text { Uy tín thương hiệu } \\
\rightarrow \text { Nhận diện thương hiệu }\end{array}$ & 0.254 & 0.075 & 3.106 & 0.002 & Chấp nhận \\
\hline H3 & $\begin{array}{l}\text { Lợi ích xã hội thương hiệu } \\
\rightarrow \text { Nhận diện thương hiệu }\end{array}$ & 0.285 & 0.071 & 3.803 & 0.000 & Chấp nhận \\
\hline $\mathrm{H} 4$ & $\begin{array}{l}\text { Sự thân thiện thương hiệu } \\
\rightarrow \text { Nhận diện thương hiệu }\end{array}$ & 0.233 & 0.073 & 3.136 & 0.002 & Chấp nhận \\
\hline H5 & $\begin{array}{l}\text { Nhận diện thương hiệu } \\
\rightarrow \text { Truyên miệng }\end{array}$ & 0.515 & 0.077 & 7.113 & 0.000 & Chấp nhận \\
\hline
\end{tabular}

Nguồn: Kết quả xử lý dữ liệu từ điều tra của nhóm tác giả

\subsection{Hàm ý cho nhà quản trị}

Thứ nhất, trong các yếu tố ảnh hưởng đến nhận diện thương hiệu điện thoại di động, lợi ích xã hội thương hiệu có ảnh hưởng lớn nhất, tiếp đến là uy tín thương hiệu, sau đó là sự thân thiện thương hiệu và cuối cùng là yếu tố sự khác biệt thương hiệu. Đây là cơ sở cho nhà tiếp thị xác định thứ tự ưu tiên khi xây dựng sự nhận diện thương hiệu. Đối với thành phần có ảnh hưởng quan trọng nhất là lợi ích xã hội thương hiệu, nhà tiếp thị cần thúc đẩy tương tác giữa thương hiệu và người tiêu dùng cũng như tương tác giữa những người tiêu dùng với nhau. Tương tác giữa thương hiệu và người tiêu dùng có thể được kích thích qua tương tác trực tuyến (website, email, mạng xã hội, ...) hoặc tương tác ngoại tuyến (tổ chức sự kiện giới thiệu sản phẩm mới, tạo điều kiện cho khách hàng góp ý, ...). Để tăng tương tác giữa những người tiêu dùng, cần thành lập cộng đồng thương hiệu để họ giao lưu, chia sẻ tình yêu thương hiệu và sử dụng KOLs (Key Opinion Leaders) tạo sức ảnh hưởng đối với nhóm khách hàng mục tiêu, vốn là những người hâm mộ, thành viên trong cộng đồng, lĩnh vực KOLs đang hoạt động. Đối với uy tín thương hiệu, nhà quản trị cần tập trung duy trì và cải tiến chất lượng sản phẩm, đảm bảo chất lượng đồng bộ và thực hiện đúng những chính sách đã cam kết với khách hàng. Ngoài ra, cần xây dựng chiến 
lược truyền thông phù hợp nhằm mở rộng uy tín thương hiệu, ví dụ, công bố giải thưởng, chứng nhận thương hiệu đạt được hay đánh giá tốt của khách hàng. Đối với sự thân thiện thương hiệu, nhà quản trị cần thực hiện truyền thông trong và ngoài doanh nghiệp nhằm xây dựng hình ảnh thân thiện với khách hàng. Sự thân thiện phải được thể hiện qua đội ngũ nhân viên nhiệt tình, tận tâm, song song đó, nhà quản trị cần cân nhắc thực thi trách nhiệm xã hội của doanh nghiệp. Các chiến lược tiếp thị phải nhấn mạnh vào cảm xúc, truyền tải giá trị thương hiệu mang lại cho xã hội nhằm thuyết phục khách hàng nghĩ về thương hiệu một cách thiện cảm. Cuối cùng, yếu tố Sự khác biệt thương hiệu có thể được tạo ra bởi thiết kế, tính năng của sản phẩm, chiến lược tiếp thị và các dịch vụ đặc biệt mà thương hiệu cung cấp cho khách hàng. Nhà tiếp thị cần lưu ý nền tảng của sự khác biệt phải xuất phát từ mục đích nâng cao cá tính và sự thỏa mãn của khách hàng, không mang hàm ý tiêu cực hoặc gây rắc rối, phiền phức cho họ.

Thứ hai, nghiên cứu cho thấy Sự khác biệt thương hiệu, lợi ích xã hội thương hiệu và sự thân thiện thương hiệu có tác động cùng chiều đến nhận diện thương hiệu của người tiêu dùng, điều này phù hợp với kết quả của Sauer và cộng sự (2012). Kết quả nhận diện thương hiệu tác động cùng chiều với Truyền miệng tương đồng với kết quả của Kuenzel và Halliday (2008), Tuškej và cộng sự (2013). Mối quan hệ giữa uy tín thương hiệu và nhận diện thương hiệu cũng được ủng hộ bởi Kuenzel và Halliday (2008). Điều này giúp nhà quản trị nhận ra: Uy tín thương hiệu sẽ tác động mạnh hơn đến Nhận diện thương hiệu khi sản phẩm có giá trị cao và nhà quản trị cần căn cứ vào đặc tính của thương hiệu để đưa ra chiến lược tiếp thị phù hợp cho từng thị trường mục tiêu.

Thứ ba, kết quả cho thấy có mối quan hệ cùng chiều nhận diện thương hiệu và truyền miệng. Điều này cho thấy nếu một thương hiệu có sự khác biệt, có uy tín, đem lại lợi ích xã hội và tạo thiện cảm đối với người dùng nhưng người dùng không tìm thấy điểm chung, không tin vào thương hiệu, thương hiệu không mang lại ý nghĩa cho cuộc sống của họ thì họ sẽ không giới thiệu, nói tốt hay khuyến khích người khác mua thương hiệu đó. Hơn nữa, nhà quản trị phải thường xuyên thăm dò ý kiến nhằm cập nhật xu hướng của khách hàng và căn cứ vào môi trường bên trong cũng như bên ngoài để ra quyết định đa dạng hóa sản phẩm. Ngoài ra, khi khách hàng nhận diện và gắn bó với thương hiệu thì những động thái của thương hiệu đi ngược lại sự tin tưởng của họ sẽ gây tổn thương cho quan hệ khách hàng - thương hiệu, tác động tiêu cực đến hành vi truyền miệng của khách hàng.

\section{Hạn chế và hướng nghiên cứu tiếp theo}

Thứ nhất, đề tài chỉ tập trung nghiên cứu tác động của các tiền tố sự khác biệt thương hiệu, uy tín thương hiệu, lợi ích xã hội của thương hiệu và sự thân thiện thương hiệu đến nhận diện thương hiệu. Thực tế, các yếu tố khác như truyền thông công ty etc., cũng có ảnh hưởng đến nhận diện thương hiệu của người tiêu dùng. Ngoài ra hậu tố của nhận diện thương hiệu không chỉ có hành vi truyền miệng mà còn có hành vi mua lại. Do vậy, các nghiên cứu tiếp theo có thể mở rộng thêm các yếu tố này để có được một mô hình toàn diện hơn.

Thứ hai, nghiên cứu này chỉ kiểm định tác động của giới tính lên quan hệ giữa các tiền tố, hậu tố của nhận diện thương hiệu. Các nghiên cứu sau có thể xem xét ảnh hưởng của các biến định tính khác như độ tuổi, thu nhập hoặc biến đặc điểm tính cách, etc.

Thứ ba, đề tài chỉ tập trung nghiên cứu nhận diện thương hiệu của sản phẩm hữu hình điện thoại di động, vì vậy, các nghiên cứu tiếp theo có thể mở rộng sang các sản phẩm hữu hình khác hoặc triển khai trong lĩnh vực dịch vụ.

Cuối cùng, nghiên cứu mang tính tổng quát cho thị trường điện thoại di động chứ chưa đi sâu vào từng thương hiệu cụ thể như Iphone, Samsung, Oppo, etc. Các nghiên cứu sau có thể khai thác từng thương hiệu cụ thể để đưa ra hàm ý quản trị thực tiễn hơn. 


\section{Tài liệu tham khảo}

Aaker, J., Vohs, K., \& Mogilner, C. (2010). Non-profits are seen as warm and for-profits as competent: Firm stereotypes matter. Journal of Consumer Research, 37(2), 224-237.

Acharya, A. (2020). The impact of brand familiarity, customer brand engagement and selfidentification on word-of-mouth. South Asian Journal of Business Studies, 10(1), 29-48.

Ahearne, M., Bhattacharya, C. B., \& Gruen, T. (2005). Antecedents and consequences of customer-company identification: Expanding the role of relationship marketing. Journal of Applied Psychology, 90(3), 574-585.

Alden, D. L., Steenkamp, J. B. E. M., \& Batra, R. (1999). Brand positioning through advertising in Asia, North America, and Europe: The role of global consumer culture. Journal of Marketing, 63(1), 75-87.

Ashforth, B. E., \& Mael, F. (1989). Social identity theory and the organization. Academy of Management Review, 14(1), 20-39.

Berger, J., \& Heath, C. (2007). Where consumers diverge from others: Identity signaling and product domains. Journal of Consumer Research, 34(2), 121-134.

Bhattacharya, C. B., \& Sen, S. (2003). Consumer-company identification: A framework for understanding consumers' relationships with companies. Journal of Marketing, 67(2), 76-88.

Brewer, M. B. (1991). The social self: On being the same and different at the same time. Personality and Social Psychology Bulletin, 17(5), 475-482.

Brown, S., Kozinets, R. V., \& Sherry, J. F., Jr. (2003). Teaching old brands new tricks: Retro branding and the revival of brand meaning. Journal of Marketing, 67(3), 19-33.

Cheung, M. L., Pires, G., \& Rosenberger, P. J. (2020). The influence of perceived social media marketing elements on consumer-brand engagement and brand knowledge. Asia Pacific Journal of Marketing and Logistics, 32(3), 695-720.

Coelho, P. S., Rita, P., \& Santos, Z. R. (2018). On the relationship between consumer-brand identification, brand community, and brand loyalty. Journal of Retailing and Consumer Services, 43, 101-110.

Diamond, N., Sherry, J. F., Jr., Muniz, A. M., Jr., McGrath, M. A., Kozinets, R. V., \& Borghini, S. (2009). American girl and the brand Gestalt: Closing the loop on sociocultural branding research. Journal of Marketing, 73(3), 118-134.

Dittmar, H. (2005). Compulsive buying-a growing concern? An examination of gender, age, and endorsement of materialistic values as predictors. British Journal of Psychology, 96(4), 467-491.

Dittmar, H., Beattie, J., \& Friese, S. (1995). Gender identity and material symbols: Objects and decision considerations in impulse purchases. Journal of Economic Psychology, 16 (3), 491-511.

Dutton, J. E., Dukerich, J. M., \& Harquail, C. V. (1994). Organizational images and member identification. Administrative Science Quarterly, 39(2), 239-263.

Elbedweihy, A. M., \& Jayawardhena, C. (2014). Consumer-brand identification: A social identity based review and research directions. The Marketing Review, 14(2), 205-228.

Ellis, B. T. (2000). The development, psychometric evaluation, and validation of a customer loyalty scale (Bachelor's thesis). Southern Illinois University, Carbondale, Illinois, USA.

Fiske, S. T., Cuddy, A. J. C., Glick, P., \& Xu, J. (2002). A model of (often mixed) stereotype content: Competence and warmth respectively follow from perceived status and competition. Journal of Personality and Social Psychology, 82(6), 878-902. 
Fornell, C., \& Larcker, D. F. (1981). Evaluating structural equation models with unobservable variables and measurement error. Journal of Marketing Research, 18(1), 39-50.

Fournier, S. (1998). Consumers and their brands: Developing relationship theory in consumer research. Journal of Consumer Research, 24(4), 343-373.

Ghorbanzadeh, D., Saeednia, H., \& Rahehagh, A. (2020). Antecedents and consequences of brand passion among young smartphone consumers: Evidence of Iran. Cogent Business \& Management, 7(1), 1-17.

Gioia, D. A., Schultz, M., \& Corley, K. G. (2000). Organizational identity, image and adaptive instability. Academy of Management Review, 25(1), 63-81.

Han, S. H., Ekinci, Y., Chen, C. H. S., \& Park, M. K. (2020). Antecedents and the mediating effect of customer-restaurant brand identification. Journal of Hospitality Marketing \& Management, 29(2), 202-220.

Hasan, B. (2010). Exploring gender differences in online shopping attitude. Computers in Human Behavior, 26(4), 597-601.

Homburg, C., Wieseke, J., \& Hoyer, W. D. (2009). Social identity and the service-profit chain. Journal of Marketing, 73, 38-54.

Hoyer, W. D., \& Brown, S. P. (1990). Effects of brand awareness on choice for a common, repeat purchase product. Journal of Consumer Research, 17(2), 141-148.

Huu Tuan (2020). Lộ diện "ông trùm” mới trên thị trường smartphone [Revealing a new "tycoon" in the smartphone market]. Retrieved April 18, 2020, from Đầu tư Online website: https://baodautu.vn/lo-dien-ong-trum-moi-tren-thi-truong-smartphone-d1 19971.html

Keller, K. L. (2003). Strategic brand management (2nd ed.). Upper Saddle River, NJ: Pearson/Prentice Hall.

Kempf, D. S., \& Palan, K. M. (2006). The effects of gender and argument strength on the processing of word-of-mouth communications. Academy of Marketing Studies Journal, 10(1), 1-18.

Kim, C. K., Han, D. C., \& Park, S. B. (2001). The effect of brand personality and brand identification on brand loyalty: Applying the theory of social identification. Japanese Psychological Research, 43(4), 195-206.

Kuenzel, S., \& Halliday, S. V. (2008). Investigating antecedents and consequences of brand identification. Journal of Product and Brand Management, 17(5), 293-304.

Kumar, V. (2020). Building customer-brand relationships through customer brand engagement. Journal of Promotion Management, 27(1), 1-27.

Kumar, V., \& Kaushik, A. K. (2018). Destination brand experience and visitor behavior: The mediating role of destination brand identification. Journal of Travel \& Tourism Marketing, 35(5), 649-663.

Lam, S. K., Ahearne, M., Mullins, R., Hayati, B., \& Schillewaert, N. (2013). Exploring the dynamics of antecedents to customer-brand identification with a new brand. Journal of the Academy of Marketing Science, 41(2), 234-252.

Lam, S. K., Ahearne, M., Hu, Y., \& Schillewaert, N. (2010). Resistance to brand switching when a radically new brand is introduced: A social identity theory perspective. Journal of Marketing, 74(6), 128-146.

McAlexander, J. H., Schouten, J. W., \& Koenig, H. F. (2002). Building brand community. Journal of Marketing, 66(1), 38-54.

Muniz, A. M., Jr., \& O’Guinn, T. C. (2001). Brand community. Journal of Consumer Research, 27(4), 412-432. 
Muniz, A., \& Schau, H. J. (2005). Religiosity in the abandoned apple newton brand community. Journal of Consumer Research, 31(4), 737-747.

Nyadzayo, M. W., Leckie, C., \& Johnson, L. W. (2020). The impact of relational drivers on customer brand engagement and brand outcomes. Journal of Brand Management, 1-18.

O’Guinn, T. C., \& Muniz, A. M., Jr. (2009). Collective brand relationships. In D. J. MacInnis, C. W. Park, \& J. R. Priester (Eds.), Handbook of brand relationships (pp. 173-194). Armonk, NY: M.E. Sharpe.

Park, C. W., MacInnis, D. J., Priester, J., Eisingerich, A. B., \& Iacobucci, D. (2010). Brand attachment and brand attitude strength: Conceptual and empirical differentiation of two critical brand equity drivers. Journal of Marketing, 74(6), 1-17.

Rather, R. A., Tehseen, S., Itoo, M. H., \& Parrey, S. H. (2019). Customer brand identification, affective commitment, customer satisfaction, and brand trust as antecedents of customer behavioral intention of loyalty: An empirical study in the hospitality sector. Journal of Global Scholars of Marketing Science, 29(2), 196-217.

Sihvonen, J. (2019). Understanding the drivers of consumer-brand identification. Journal of Brand Management, 26(5), 583-594.

Sauer, N., Ratneshwar, S., \& Sen, S. (2012). Drivers of consumer-brand identification. International Journal of Research in Marketing, 29(4), 406-418.

Tajfel, H., \& Turner, J. (1986). The social identity theory of intergroup behavior. In S. Stephen Worchel \& W. G. Austin (Eds.), Social psychology of intergroup relations (pp. 7-24). Chicago, IL: Nelson- Hall.

Thompson, C. J., Rindfleisch, A., \& Arsel, Z. (2006). Emotional branding and the strategic value of the Doppelgänger brand image. Journal of Marketing, 70(1), 50-64.

Thomson, M., MacInnis, D. J., \& Park, C. W. (2005). The ties that bind: Measuring the strength of consumers' emotional attachment to brands. Journal of Consumer Psychology, 15(1), 77-91.

Truch, E. (2006). Lean consumption and its influence on brand. Journal of Consumer Behaviour, $5(1), 157-165$.

Truong, Y., McColl, R., \& Kitchen, P. J. (2009). New luxury brand positioning and the emergence of masstige brands. Journal of Brand Management, 16(5), 375-382.

Thanh Truc (2018). Người Việt chi gần 20.000 tỉ mua smartphone trong quý 1 - 2018 [Vietnamese people spent nearly 20,000 billion buying smartphones in the first quarter of 2018]. Retrieved April 06, 2018, from Tuổi trẻ Online website: https://congnghe.tuoitre.vn/thi-truong/nguoi-viet-chi-gan-20000-ti-mua-smartphone-trongquy-12017-1312164.htm

Tuškej, U., Golob, U., \& Podnar, K. (2013). The role of consumer-brand identification in building brand relationships. Journal of Business Research, 66, 53-59.

Yeh, C. H., Wang, Y. S., \& Yieh, K. (2016). Predicting smartphone brand loyalty: Consumer and consumer-brand identification perspectives. International Journal of Information Management, 36, 245-257.

Zhang, M., Li, L., Ye, Y., Qin, K., \& Zhong, J. (2020). The effect of brand anthropomorphism, brand distinctiveness, and warmth on brand attitude: A mediated moderation model. Journal of Consumer Behaviour, 19(5), 523-536.

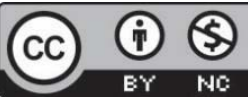

Creative Commons Attribution-NonCommercial 4.0 International License. 